\title{
Proximity in arrangements of algebraic sets
}

\author{
J.H. Rieger* \\ Max-Planck-Institut für Informatik \\ Im Stadtwald, D-66123 Saarbrücken, FRG
}

\begin{abstract}
Let $X$ be an arrangement of $n$ algebraic sets $X_{i}$ in $d$-space, where the $X_{i}$ are either parameterized or zero-sets of dimension $0 \leq m_{i} \leq d-1$. We study a number of decompositions of $d$-space into connected regions in which the distance-squared function to $X$ has certain invariances. These decompositions can be used in the following of proximity problems: given some point, find the $k$ nearest sets $X_{i}$ in the arrangement, find the nearest point in $X$ or (assuming that $X$ is compact) find the farthest point in $X$ and hence the smallest enclosing $(d-1)$-sphere. We give bounds on the complexity of the decompositions in terms of $n, d$, and the degrees and dimensions of the algebraic sets $X_{i}$.
\end{abstract}

* Address for correspondence (from March 1996 on):

ICMSC, Univ. Sao Paulo, Caixa Postal 668, 13560 Sao Carlos - SP, Brazil

E-mail: rieger@xavante.icmsc.sc.usp.br 


\section{Introduction}

Let $X$ be the union of $n$ algebraic sets $X_{i}$ of dimension $0 \leq m_{i} \leq d-1$ in $d$-space which are either defined by parametrizations or, more general, as zero-sets. Given a point $p \in \mathbb{R}^{d}$ with rational or, more general, with algebraic number coordinates and a set of defining polynomials of $X$ with rational coefficients, we would like to do the following:

1. find the $k$ nearest sets $X_{i}$;

2. find the nearest point in $X$;

3. and, provided that $X$ is compact, find the farthest point in $X$ (and hence the smallest sphere with centre $p$ enclosing $X$ ).

For all of these proximity problems it is convenient to decompose $d$-space into certain connected regions, depending on $X$, in which the distance-squared function to $X$ has certain invariances. A number of such decompositions are possible. Some decompositions have many invariants but also many regions, and it is of interest to bound the number of regions in terms of $n, d$, and the degrees and dimensions of the algebraic sets $X_{i}$. For example, the coarsest decomposition considered below consists of the first-order Voronoi regions and the finest consists of the regions in the complement of the bifurcation set of the family of all distance-squared functions on $X$. However, all the decompositions studied here have the property that the proximity problems above can be solved in $O(\log n)$ time (discarding the preprocessing time for constructing the decomposition). Decompositions of $d$-space into regions made of points having certain proximity properties with respect to some collection of submanifolds of $\mathbb{R}^{d}$ have been studied both in computational geometry and in singularity theory, but there hasn't been much interaction between these fields.

Most of the works in computational geometry either consider the classical, first-order, Voronoi diagram of sets of isolated points or extensions to arrangements of linear subspaces of $\mathbb{R}^{d}$. The relation between higherorder Voronoi diagrams in $\mathbb{R}^{d}$ and arrangements in $\mathbb{R}^{d+1}$ is investigated in Edelsbrunner and Seidel [8]. A few works also consider Voronoi diagrams of arrangements of curved objects. First-order Voronoi diagrams of disjoint convex semi-algebraic sites in $d$-space are studied in the book of Sharir and Agarwal [16]. Alt and Schwarzkopf [1] study first-order Voronoi diagrams of parameterized (semi-algebraic) curve-segments and points in the plane. These authors are also interested in the local geometry of Voronoi edges: for example, they point out that end-points of self-Voronoi-edges (in the singularity theory literature known as symmetry sets) correspond to centres of osculating circles at curvature extrema of a planar curve and also to a cusp singularity of the evolute (or focal set). The local geometry of such 
symmetry sets and of evolutes has been studied in great detail in a number of singularity theory works.

One of the main topics of singularity theory is the classification of stable and unstable singularities of functions and maps, and of the bifurcation sets in the parameter space of families of functions and maps. The bifurcation set of a family of functions $F: \mathbb{R}^{d} \times \mathbb{R}^{m} \rightarrow \mathbb{R}^{d} \times \mathbb{R},(p, x) \mapsto(p, f(p, x))$ consists of all points $p$ in parameter space $\mathbb{R}^{d}$ for which the function $x \mapsto f(p, x)$ has an unstable (degenerate) singularity. The family of distance-squared functions from any point $p \in \mathbb{R}^{d}$ to a parameterized $m$-dimensional surface $X$ in $d$-space is a particular example of such a family, and the bifurcation set of this family is precisely the union of the evolute and the symmetry set of $X$. Porteous $[11,12]$ has used the classification of families of functions by Thom (from the early 1960s) to study the relation between the geometry of evolutes and the curvature of surfaces. Bruce et al. [4] have classified the singularities of symmetry sets of planar curves and of surfaces and spacecurves in $\mathbb{R}^{3}$, see also the recent paper by Bruce [3]. Symbolic algorithms for computing bifurcation sets of families of projection maps have been studied by Rieger in $[14,15]$ and these algorithms can be also used, with some minor modifications, to compute other bifurcation sets, such as evolutes and symmetry sets.

\subsection{Assumptions and some notation}

Let $X:=\bigcup X_{i} \subset \mathbb{R}^{d}$ be a collection of $n$ closed algebraic sets $X_{i}$ and set $m_{i}:=\operatorname{dim} X_{i}\left(0 \leq m_{i} \leq d-1\right)$ and $m:=\sup m_{i}$. For parameterized algebraic $m_{i}$-surfaces $x \mapsto X_{i}(x)$ we denote the maximal degree of the $d$ component functions of $X_{i}(x)$ by $\delta_{i}$, and set $\delta:=\sup \delta_{i}(1 \leq i \leq n)$. For the more general case of zero-sets $X_{i}=h_{i}^{-1}(0)$, where $h_{i}:=\left(h_{i}^{1}, \ldots, h_{i}^{d-m_{i}}\right)$ : $\mathbb{R}^{d} \rightarrow \mathbb{R}^{d-m_{i}}$, we assume that $X_{i}$, or rather its complexification, is a complete intersection (i.e. its codimension is equal to the number of defining equations) and set $\Delta_{i}:=\prod_{j} \operatorname{deg} h_{i}^{j}=\operatorname{deg} X_{i}$ and $\Delta:=\sup \Delta_{i}$ (geometrically, $\operatorname{deg} X_{i}$ is the number of real and complex intersection points of $X_{i}$ and a "generic" linear subspace of $\mathbb{R}^{d}$ of dimension $d-m_{i}$ ).

The following notation will be used in this paper: $Z(I)$ denotes the zeroset of an ideal $I, I(Z)$ the ideal of polynomials vanishing on $Z, I: J$ the ideal quotient and $\mathrm{cl} Z$ denotes the closure of the set $Z$. The components of a vector $x=\left(x^{1}, \ldots, x^{d}\right)$ are denoted by superscripts, so that subscripts can be used to enumerate elements of sets; and $\left(x^{d}\right)^{3}$ denotes the third power of the $d$ th component. A function $f: \mathbb{R}^{d} \rightarrow \mathbb{R}, x:=\left(x^{1}, \ldots, x^{d}\right) \mapsto f(x)$ has an $A_{k}$-singularity at $x=0$ if there exists a smooth coordinate change $h: \mathbb{R}^{d} \rightarrow \mathbb{R}^{d}$, defined in the neighborhood of $x=0$, such that $f \circ h(x)=$ $c+\left(x^{1}\right)^{k+1}+\sum_{i=2}^{d} \epsilon_{i}\left(x^{i}\right)^{2}$, where $c$ is some constant and $\epsilon_{i}= \pm 1$. (Also, the reader familiar with singularity theory will note that we abuse the notation $A_{\geq k}$ slightly: here it denotes all classes of singularities in the closure of the 
$A_{k}$ orbit, not just the $A_{\geq k}$ orbits.)

\subsection{Content of following sections}

In Section 2 we study the bifurcation set $\mathcal{B}$ of the family of distance-squared functions on an arrangement of $m_{i}$-surfaces in $\mathbb{R}^{d}$ which are parameterized by polynomial maps. In particular, we give bounds for the number of regions in the complement of $\mathcal{B}$ and describe certain invariants which characterize these regions.

In Section 3 we consider the more general case of arrangements of algebraic zero-sets $X_{i}$ (note that most zero-sets do not have a global parametrization given as the image of some polynomial map). For zero-sets we exploit the geometric characterization of the singularities of the distance-squared function in terms of the contact order (or intersection multiplicity) of $X_{i}$ with certain $\left(d-m_{i}\right)$-spheres, where $m_{i}=\operatorname{dim} X_{i}$. This avoids the problem of finding local parametrizations of the $X_{i}$ given by analytic maps (working with polynomials is much more convenient). The more classical case of contact between hypersurfaces $X_{i}$ and osculating circles is treated in Subsection 3.1; the more complicated case of contact between algebraic sets $X_{i}$ of codimension $d-m_{i} \geq 2$ and $\left(d-m_{i}\right)$-spheres is studied in Subsection 3.2.

In Section 4 we give an outline of algorithms for determining the regions in the complement of the bifurcation set $\mathcal{B}$ and certain other decompositions of $d$-space into coarser regions. Using these decompositions, we describe solutions to the proximity problems 1 to 3 stated at the beginning of this introduction.

In Section 5 we present a few examples of these decompositions for curves and points in the plane, which have been computed with the methods described in Section 4.

Finally, in Section 6, we compare the combinatorial complexities of the set of connected regions of $\mathbb{R}^{d} \backslash \mathcal{B}$ and of $k$ th-order Voronoi diagrams.

\section{The complement of the bifurcation set $\mathcal{B}$ of a family of distance-squared functions}

In the present section the algebraic $m_{i}$-surfaces $X_{i}$ of the arrangement are parameterized by polynomial maps $x \mapsto X_{i}(x)$, where $x=\left(x^{1}, \ldots, x^{m_{i}}\right) \in$ $\mathbb{R}^{m_{i}}$. The necessary modifications in the (more general) case of zero-sets will be briefly sketched in Section 3. The family of distance-squared functions on $X_{i}$ is defined by

$$
F_{i}: \mathbb{R}^{d} \times \mathbb{R}^{m_{i}} \rightarrow \mathbb{R}^{d} \times \mathbb{R}, \quad(p, x) \mapsto\left(p, f_{i}(p, x):=\left\|X_{i}(x)-p\right\|^{2}\right) .
$$

Recall that an element of this $d$-parameter family of functions in $m_{i}$ variables is a Morse function if its critical points are non-degenerate (i.e. the 
corresponding matrix of second derivatives has maximal rank) and have distinct critical values. The bifurcation set $\mathcal{B}_{i} \subset \mathbb{R}^{d}$ of the family $F_{i}$ is the set of "bad" parameters $p$ for which $x \mapsto f_{i}(p, x)$ fails to be a Morse function. The set $\mathcal{B}_{i}$ is the union of the local bifurcation set

$$
\mathcal{E}_{i}:=\left\{p \in \mathbb{R}^{d}: \exists x: d f_{i}(p, x)=0, \text { rank } d^{2} f_{i}(p, x)<m_{i}\right\}
$$

and the level bifurcation set

$$
\mathcal{S}_{i}:=\operatorname{cl}\left\{p \in \mathbb{R}^{d}: \exists x \neq \bar{x}: d f_{i}(p, x)=d f_{i}(p, \bar{x})=0, f_{i}(p, x)=f_{i}(p, \bar{x})\right\} .
$$

(The notation $\mathcal{E}_{i}$ and $\mathcal{S}_{i}$ indicates that, from a classical differential geometry point of view, the local and level bifurcation sets are evolutes and symmetry sets, respectively - see Section 3.)

The bifurcation set $\mathcal{B}$ of the arrangement associated to $X=\bigcup X_{i}$ is the union of the bifurcation sets $\mathcal{B}_{i}$ of the $X_{i}$ and the following inter-surface level bifurcation sets

$$
\mathcal{S}_{i, j}:=\left\{p \in \mathbb{R}^{d}: \exists x, \bar{x}: d f_{i}(p, x)=d f_{j}(p, \bar{x})=0, f_{i}(p, x)=f_{j}(p, \bar{x})\right\},
$$

that is

$$
\mathcal{B}:=\bigcup_{1 \leq i \leq n} \mathcal{E}_{i} \cup \bigcup_{1 \leq i \leq n} \mathcal{S}_{i} \cup \bigcup_{1 \leq i<j \leq n} \mathcal{S}_{i, j}
$$

This definition of $\mathcal{B}$ asssumes that $1 \leq \operatorname{dim} X_{i} \leq d-1$, but it can be easily extended to include isolated points $X_{i}=\left\{q_{i}\right\}$. For a point $q_{i}$ the sets $\mathcal{E}_{i}$ and $\mathcal{S}_{i}$ are defined to be empty, for a point pair $q_{i}, q_{j}$ the set $\mathcal{S}_{i, j}$ is defined to be the hyperplane perpendicular to $q_{j}-q_{i}$ through $\left(q_{i}+q_{j}\right) / 2$ and for surface-point pairs $X_{i}\left(\operatorname{dim} X_{i} \geq 1\right), X_{j}=\left\{q_{j}\right\}$ we define

$$
\mathcal{S}_{i, j}:=\left\{p \in \mathbb{R}^{d}: \exists x: d f_{i}(p, x)=0, f_{i}(p, x)=\left\|q_{j}-p\right\|^{2}\right\} .
$$

The definitions of the local bifurcation sets $\mathcal{B}_{i}$ and of the inter-surface level bifurcation sets $\mathcal{S}_{i, j}$ are fairly straightforward from a computational point of view. The definition of the intra-surface level bifurcation sets $\mathcal{S}_{i}$ is less straightforward: the inequalities $x \neq \bar{x}$, together with the defining equations appearing in the definition, yield semi-algebraic sets $\mathcal{S}_{i}^{\prime} \subset \mathbb{R}^{d} \times \mathbb{R}^{2 m_{i}}$ which are not closed. It is, however, possible to close-11p the sets $\mathcal{S}_{i}^{\prime}$ by adding a set of boundary points $\partial \mathcal{S}_{i}^{\prime}$ on the diagonal $\{x=\bar{x}\} \subset \mathbb{R}^{2 m_{i}}$ (see below). Furthermore, the closed sets $\tilde{\mathcal{S}}_{i}:=\mathcal{S}_{i}^{\prime} \cup \partial \mathcal{S}_{i}^{\prime}$ can be defined by polynomial equations (inequations are not required), which is a big advantage from a computational algebra point of view. 
2.1 Proposition. (i) For all $(p, x) \in \partial \mathcal{S}_{i}^{\prime}$, the distance-squared function $x \mapsto f_{i}(p, x)$ has an $A_{>3}$-singularity at $x$. This implies that $\pi\left(\partial \mathcal{S}_{i}^{\prime}\right) \subset \mathcal{B}_{i}$, where $\pi: \mathbb{R}^{d} \times \mathbb{R}^{2 m_{i}} \rightarrow \mathbb{R}^{d}$ denotes the projection onto the first factor. (ii) The degree of $\partial \mathcal{S}_{i}^{\prime}$ is of order $\delta_{i}^{2 m_{i}+1}$ and that of $\bigcup \partial \mathcal{S}_{i}^{\prime}$ of order $n \cdot \delta^{2 m+1}$.

Proof. (i) Replace the coordinates $(x, \bar{x}) \in \mathbb{R}^{2 m_{i}}$ by $(x, y)$, where $y:=$ $\left(\lambda, a^{1}, \ldots, a^{m_{i}-1}\right)$ and $\bar{x}:=x+\lambda \cdot\left(a^{1}, \ldots, a^{m_{i}-1}, 1\right)$. The condition $x \neq \bar{x}$ in the old coordinate system corresponds, roughly speaking, to $\lambda \neq 0$ in the new one (note that $\bar{x}-x \in \mathbb{P}^{m_{i}-1} \backslash\left\{a^{m_{i}}=0\right\}$ - in general two such affine charts are required in order to obtain all possible points $\bar{x}$ ). We then claim that the sets $\mathcal{S}_{i}$ can be defined by the following three equations (omitting the inequality $\lambda \neq 0$ ): by $d f_{i}(p, x)=0$ (as before) and by

$$
U_{i}(p, x, y):=\lambda^{-1}\left(d f_{i}(p, \bar{x})-d f_{i}(p, x)\right)=0
$$

and

$$
\begin{aligned}
V_{i}(p, x, y):=\lambda^{-3}\left(f_{i}(p, \bar{x})\right. & -f_{i}(p, x)-\lambda d f_{i}(p, x) \\
& \left.-\frac{\lambda^{2}}{2}\left\langle U_{i},\left(a^{1}, \ldots, a^{d-2}, 1\right)\right\rangle\right)=0 .
\end{aligned}
$$

It is easy to see that, away from the diagonal $\{x=\bar{x}\}, d f_{i}(p, x)=U_{i}(p, x, y)=$ $V_{i}(p, x, y)=0$ and the original system $d f_{i}(p, x)=d f_{i}(p, \bar{x})=f_{i}(p, x)-$ $f_{i}(p, \bar{x})=0$ define the same zero-sets $\mathcal{S}_{i}^{\prime} \subset \mathbb{R}^{d} \times \mathbb{R}^{2 m_{i}} \backslash\{x=\bar{x}\}$. Furthermore, the right-hand-sides of $U_{i}$ and $V_{i}$ are divisible by $\lambda$ and $\lambda^{3}$ (by Taylor's theorem), hence $d f_{i}(p, x)=U_{i}(p, x, y)=V_{i}(p, x, y)=0$ defines a closed algebraic variety $\tilde{\mathcal{S}}_{i}:=\mathcal{S}_{i}^{\prime} \cup \partial \mathcal{S}_{i}^{\prime} \subset \mathbb{R}^{d} \times \mathbb{R}^{2 m_{i}}$.

In fact, $\tilde{\mathcal{S}}_{i}$ is the smallest closed set containing $\mathcal{S}_{i}^{\prime}$ and the boundary $\partial \mathcal{S}_{i}^{\prime}$ of $\mathcal{S}_{i}^{\prime}$ in $\tilde{\mathcal{S}}_{i}$ corresponds to $A_{\geq 3}$-singularities of the distance-squared function $x \mapsto f_{i}(p, x)$. Taking the limit as $\lambda \rightarrow 0$ (corresponding to the diagonal $\{x=\bar{x}\})$ we get the following system:

$$
\begin{aligned}
d f_{i}(p, x) & =0 \\
\lim _{\lambda \rightarrow 0} U_{i}=\frac{1}{2} d^{2} f_{i}(p, x)\left(\left(a^{1}, \ldots, a^{m_{i}-1}, 1\right)\right) & =0 \\
\lim _{\lambda \rightarrow 0} V_{i}=\frac{1}{6} d^{3} f_{i}(p, x)\left(\left(a^{1}, \ldots, a^{m_{i}-1}, 1\right)^{3}\right) & =0 .
\end{aligned}
$$

This system "recognizes" an $A_{>3}$-singularity of $x \mapsto f_{i}(p, x)$ at $x$ - the condition for an $A_{\geq 3}$-singularity is precisely that $d f_{i}=0$ and $d^{2} f_{i}(v)=0$, $d^{3} f_{i}\left(v^{3}\right)=0$ for some non-zero vector $v$ (see, for example, Porteous [13], p. $397)$.

(ii) The degree of the variety $\partial \mathcal{S}_{i}^{\prime}$ defined by the above "limiting system" is at most of order $\delta_{i}^{2 m_{i}+1}$ (by Bezout's theorem), so that the degree of the 
union of $n$ such varieties is of order $\sum_{i=1}^{n} \delta_{i}^{2 m_{i}+1} \leq n \cdot \delta^{2 m+1}$.

2.2 Corollary. The level-bifurcation set of the distance squared-functions of an arrangement of $n$ plane algebraic curves of maximal degree $\delta$ has at most $O\left(n \cdot \delta^{3}\right)$ endpoints. (Actually, there are at most $O(n \cdot \delta)$ endpoints.)

Proof. For $m=1$ and $d=2$, the set $\bigcup \partial \mathcal{S}_{i}^{\prime}$ consists of isolated points $\left(p_{l}, x_{l}\right) \in \mathbb{R}^{2} \times \mathbb{R}$ (this can be checked by a simple dimensional argument), and there are at most $O\left(n \cdot \delta^{3}\right)$ such endpoints by the proposition above. The projections $p_{l}$ of these points into the plane are possible endpoints of the level-bifurcation set $\mathcal{S}=\bigcup \mathcal{S}_{i}$. (However, their projections $x_{l}$ onto $\mathbb{R}$ correspond to curvature extrema of $X_{i}$ and each curvature extremum corresponds to one endpoint. But $X=\bigcup X_{i}$ has at most $O(n \cdot \delta)$ curvature extrema.)

2.3 Proposition. For all points $p$ in a single connected region of $\mathbb{R}^{d} \backslash$ $\bigcup \mathcal{E}_{i}$ the collection of distance-squared functions $\left\{x \mapsto f_{i}(p, x): 1 \leq i \leq n\right\}$ has a constant number, $c$, of critical points, where

$$
n \leq c \leq \sum_{i=1}^{n}\left(2 \delta_{i}-1\right)^{m_{i}} \sim O\left(n \cdot \delta^{m}\right) .
$$

Proof. From the definition of the local bifurcation sets $\mathcal{E}_{i}$ we see that the distance-squared functions $x \mapsto f_{i}(p, x)$ have isolated critical points (of multiplicity 1) for all $p \in \mathbb{R}^{d} \backslash \bigcup \mathcal{E}_{i}$. Each $f_{i}$ is non-negative and has degree $2 \delta_{i}$. Hence each $f_{i}$ has at least one local minimum and at most $\left(2 \delta_{i}-1\right)^{m_{i}}$ critical points - this yields the desired bounds for $c$.

REMARK. For arrangements of hypersurfaces $X_{i}$ (i.e. $m_{i}=d-1$ ) the number of critical points $c$ has the following geometrical interpretation: it is equal to the number of normal lines of $X=\bigcup X_{i}$ passing through the point $p$.

2.4 Proposition. The number of connected regions of $\mathbb{R}^{d} \backslash \mathcal{B}$ is at most of order $n^{2 d} \cdot \delta^{(2 m+1) d}$. Furthermore, let $p \in \mathbb{R}^{d} \backslash \mathcal{B}$ and let

$$
\xi_{1, \nu_{1}}(p), \xi_{2, \nu_{2}}(p), \ldots, \xi_{c, \nu_{c}}(p)
$$

denote the critical points $\xi_{l, \nu_{l}}(p)$ of the collection of distance-squared functions $x \mapsto f_{\nu_{l}}(p, x)$, where $\nu_{l} \in\{1, \ldots, n\}$, ordered by increasing distance. That is, $f_{\nu_{l}}\left(p, \xi_{l, \nu_{l}}(p)\right)<f_{\nu_{l+1}}\left(p, \xi_{l+1, \nu_{l+1}}(p)\right)$. For all points $p$ in a single connected region of $\mathbb{R}^{d} \backslash \mathcal{B}$ we have the following: (i) the numbers $\nu_{1}, \ldots, \nu_{c}$ are invariant, and (ii) the maps $p \mapsto \xi_{l, \nu_{l}}(p)$ are continuous for $1 \leq l \leq n$. 
Proof. The bifurcation set $\mathcal{B}$ is a semi-algebraic subset of a closed real algebraic set $\hat{\mathcal{B}} \subset \mathbb{R}^{d}$, and the number of connected regions cut out by $\mathcal{B}$ is less than or equal to the number of regions cut out by $\hat{\mathcal{B}}$. The number of connected regions of $\mathbb{R}^{d} \backslash \hat{\mathcal{B}}$ is equal to the $(d-1)$ st Betti number of $\hat{\mathcal{B}}$ (see below), and the desired upper bound follows at once from a result of Milnor [10] — which says that the sum of the Betti number of $\hat{\mathcal{B}}$ is of order $(\operatorname{deg} \hat{\mathcal{B}})^{d}$ - and the bound for the degree of $\hat{\mathcal{B}}$ derived below. [The reader who is familiar with some algebraic topology will note the following: if $S^{d}=\mathbb{R}^{d} \cup\{\infty\}$ and $\hat{\mathcal{B}}^{c}=\hat{\mathcal{B}} \cup\{\infty\}$ denote 1-point compactifications then the following isomorphisms of (co-)homology groups hold

$$
H_{0}\left(S^{d} \backslash \hat{\mathcal{B}}^{c}\right) \cong H^{d}\left(S^{d}, \hat{\mathcal{B}}^{c}\right) \cong H^{d}\left(\mathbb{R}^{d}, \hat{\mathcal{B}}\right),
$$

where the first isomorphism stems from the Lefschetz duality and the second is induced from the inclusion of the pair $\left(\mathbb{R}^{d}, \hat{\mathcal{B}}\right) \hookrightarrow\left(S^{d}, \hat{\mathcal{B}}^{c}\right)$. Finally, from the standard exact sequence of the pair $\left(\mathbb{R}^{d}, \hat{\mathcal{B}}\right)$, we see that $H_{d}\left(\mathbb{R}^{d}, \hat{\mathcal{B}}\right) \cong$ $H_{d-1}(\hat{\mathcal{B}})$. It now follows that the number of regions cut out by $\hat{\mathcal{B}}$ is equal to $\left.b_{d-1}(\hat{\mathcal{B}}).\right]$

We claim that the degree of $\hat{\mathcal{B}}$ is of order $n^{2} \delta^{2 m+1}$. The set $\hat{\mathcal{B}}$ is the union of $\left(\begin{array}{l}n \\ 2\end{array}\right)$ (real algebraic) sets $\hat{\mathcal{S}}_{i, j}, n$ sets $\hat{\mathcal{S}}_{i}$ and $n$ sets $\hat{\mathcal{E}}_{i}$. The orders of the degrees of the $\hat{\mathcal{S}}_{i}$ and the $\hat{\mathcal{E}}_{i}$ are lower than those of the $\hat{\mathcal{S}}_{i, j}$, hence it suffices to estimate the degree of $\hat{\mathcal{S}}_{i, j}$. So let $\tilde{\mathcal{S}}_{i, j} \subset \mathbb{R}^{d} \times \mathbb{R}^{m_{i}+m_{j}}$ be the real algebraic set defined by the defining equations of $\mathcal{S}_{i, j}$ (omitting the existential quantifier). The restriction of the projection $\pi: \mathbb{R}^{d} \times \mathbb{R}^{m_{i}+m_{j}} \rightarrow \mathbb{R}^{d}$ to $\tilde{\mathcal{S}}_{i, j}$ yields the semi-algebraic set $\mathcal{S}_{i, j}$. Complexifying the defining equations of $\tilde{\mathcal{S}}_{i, j}$ and taking the real part of the projection $\pi$ onto $\mathbb{C}^{d}$ of the resulting zero-set yields a closed real algebraic set $\hat{\mathcal{S}}_{i, j} \subset \mathbb{R}^{d}$ which contains the semi-algebraic set $\mathcal{S}_{i, j}$. Suppose that $\operatorname{codim} \hat{\mathcal{S}}_{i, j}=1$ (otherwise the complement of $\hat{\mathcal{S}}_{i, j}$ is connected, and we are done) and let $\mathcal{L} \subset \mathbb{R}^{d}$ be any line. Now there are two cases: (1) the set $A:=\pi^{-1}(\mathcal{L}) \cap \tilde{\mathcal{S}}_{i, j}$ consists of isolated points the "generic case") and (2) $\operatorname{dim} A=e>0$. Let $\bar{\pi}: \mathbb{R}^{d} \times \mathbb{R}^{m_{i}+m_{j}} \rightarrow \mathbb{R}^{m_{i}+m_{j}}$ denote the projection onto the second factor, let $\overline{\mathcal{L}} \subset \mathbb{R}^{m_{i}+m_{j}}$ be any linear subspace of codimension $e$ such that $\bar{\pi}^{-1}(\overline{\mathcal{L}})$ is not contained in $A$ and set $\bar{A}:=A \cap \bar{\pi}^{-1}(\overline{\mathcal{L}})$. The sets $A$, in case (1), and $\bar{A}$, in case (2), are discrete point sets and the restriction of $\pi$ to these point sets onto the set of intersection points $\hat{\mathcal{S}}_{i, j} \cap \mathcal{L}$ is surjective. The degree of $\hat{\mathcal{S}}_{i, j}$ is therefore bounded by the number of points of $A$ (or $\bar{A}$ in case (2)). Inspecting the defining equations of these sets, we get from Bezout's theorem that

$$
\operatorname{deg} \hat{\mathcal{S}}_{i, j} \leq\left(2 \delta_{i}-1\right)^{m_{i}} \cdot\left(2 \delta_{j}-1\right)^{m_{j}} \cdot 2 \max \left(\delta_{i}, \delta_{j}\right)
$$

(counting both real and complex roots with their multiplicities).

For the proof of the second part of the proposition, consider the following real algebraic set:

$$
\Sigma_{F_{i}}:=\left\{(p, x): d f_{i}(p, x)=0\right\} \subset \mathbb{R}^{d} \times \mathbb{R}^{m_{i}} .
$$


The set $\Sigma_{F_{i}}$ is the critical set of the family $F_{i}$ of all distance-squared functions $f_{i}$ on $X_{i}$. The fibres $\pi^{-1}(p) \cap \Sigma_{F_{i}}$ of the projection $\pi: \mathbb{R}^{d} \times \mathbb{R}^{m_{i}} \rightarrow \mathbb{R}^{d}$ correspond to the critical points of $f_{i}$ from $p$. The restriction of $\pi$ to $\Sigma_{F_{i}}$ is a covering map whose branch-locus is the (pre-image of the) evolute $\mathcal{E}_{i}$ and which is finite-to-one off the branch-locus. The number of points in each fibre $\pi^{-1}(p) \cap \Sigma_{F_{i}}$ is therefore finite and constant for all points $p$ in a connected region of $\mathbb{R}^{d} \backslash \mathcal{E}_{i}$. The same is true for the total number $c$ of critical points of a collection $\left\{f_{i}\right\}_{1 \leq i<n}$ of distance-squared functions on $X:=\bigcup X_{i}$ for all $p$ in a single connected region of $\mathbb{R}^{d} \backslash \bigcup \mathcal{E}_{i}$. Furthermore, the indices $\nu_{1}, \ldots, \nu_{c}$ are invariant within a connected region of $\mathbb{R}^{d} \backslash \cup \mathcal{E}_{i} \cup \cup \mathcal{S}_{i, j}$, because $c$ is constant and permutations of indices can only occur along the inter-surface level bifurcation sets $\mathcal{S}_{i, j}$. Finally, let $U$ be any connected region of $\mathbb{R}^{d} \backslash \bigcup \mathcal{E}_{i}$ and consider the union of the $n$ bundles $\bigcup_{i=1}^{n} \pi^{-1}(U) \cap \Sigma_{F_{i}}$. This is a semi-algebraic set consisting of $c$ disjoint components of dimension $d$, and these components are the graphs of continuous maps $h_{j}: U \rightarrow \mathbb{R}^{m_{i}}$, $1 \leq j \leq c$ (these facts are established by arguments that are quite similar to the proof of the First Main Structure Theorem in Chapter 2.2 of [2] — in fact, most stratification schemes of semi-algebraic sets seem to be based on some version of this theorem). The composition of the $h_{j}$ with the projection $\bar{\pi}: \mathbb{R}^{d} \times \mathbb{R}^{m_{i}} \rightarrow \mathbb{R}^{m_{i}}$ is a continuous map, which implies that the $c$ critical points of the collection of distance-squared functions $x \mapsto f_{i}(p, x), 1 \leq i \leq n$, vary continuously with $p \in U$. The continuity of the maps $p \mapsto \xi_{l, \nu_{l}}(p)$ for all $p$ within a single connected region of $\mathbb{R}^{d} \backslash \mathcal{B}$ then follows from the results above and the fact that the permutation of the critical points of a single function $x \mapsto f_{i}(p, x)$ can only occur on $\mathcal{S}_{i}$.

\section{Contact of $X$ with spheres and the definition of $\mathcal{B}$ for zero-sets $X$}

The fibres of the distance-squared function from a point $p \in \mathbb{R}^{d}$ are $(d-$ $1)$-spheres of varying radius $r$, given by $\left\{x \in \mathbb{R}^{d}:\|x-p\|^{2}-r^{2}=0\right\}$. The conditions for an $A_{k}$-singularity of the distance-squared function, which appear in the definition of the bifurcation set $\mathcal{B}$, can be reformulated in more geometric terms involving the contact between a family of such spheres and a collection $X=\bigcup X_{i}$ of algebraic sets. Using these more geometric conditions, we can easily define, and compute, the bifurcation set $\mathcal{B}$ in the case of algebraic sets $X_{i}$ given as zero-sets of polynomials $h_{i}^{j} \in \mathbb{Q}[x]=$ $\mathbb{Q}\left[x^{1}, \ldots, x^{d}\right], 1 \leq j \leq d-m_{i}$.

We first consider the special case of algebraic hypersurfaces (codimension 1) where the local and level bifurcation sets of the distance-squared function are the well-known evolutes and symmetry sets of classical differential geometry (Section 3.1). In Section 3.2 we consider the more general case of 
arrangements of algebraic sets $X_{i}$ of codimension $1 \leq d-m_{i} \leq d-1$ which are complete intersections (i.e. are defined by $d-m_{i}$ polynomials). Note that the case of points $X_{i}$ (of codimension $d$ ) can be handled as in Section 2.

\subsection{Arrangements of hypersurfaces, evolutes and symmetry sets}

First, recall that a hypersurface $X_{i}$ in $d$-space has $d-1$ (not necessarily distinct) principal curvatures $\kappa_{j}$ and directions $d_{j}$ which are the eigenvalues and eigendirections of the Weingarten map. (The Weingarten map $W_{p}$ : $T_{p} X_{i} \rightarrow T_{p} X_{i}, v \mapsto-\nabla_{v} N$ measures the rate of change of the normal direction $N$ along a direction $v$ in the tangent space of $X_{i}$ at $p$.) A $(d-1)$ sphere is a curvature sphere at $x \in X_{i}$ if its centre lies on the normal line through $x$ and its radius $r$ is the inverse of one of the principal curvatures of $X_{i}$ at $x$. The unique great circle in this curvature sphere whose tangent line at $x$ is oriented along the principal direction associated to $1 / r$ is an osculating circle. The evolute (or focal surface) $\mathcal{E}_{i}$ of $X_{i}$ is the locus of centres of such osculating circles and of the curvature spheres containing them (for each surface patch of $X_{i}$ there are generically $d-1$ sheets of the evolute, one for each principal curvature).

The distance-squared function from $p \in \mathbb{R}^{d}$ to $X_{i}$ has an $A_{k^{-}}$singularity $(k \geq 1)$ at $x \in \mathbb{R}^{d}$ if and only if there exists a circle with centre $p$ having $(k+1)$-point contact with $X_{i}$ at $x$. The order of contact is $\geq 2$ if $p$ lies on the normal line to $X_{i}$ at $x$ and $\geq 3$ if, in addition, the circle is an osculating circle. The local bifurcation set $\mathcal{E}_{i}$ consists of points $p$ for which the distance-squared function to $X_{i}$ has an $A_{\geq 2}$-singularity, such points are centres of osculating circles (and of curvature spheres) - the local bifurcation set $\mathcal{E}_{i}$ is therefore the evolute of $X_{i}$. The relation between singularities of the distance-squared function, normal singularities of submanifolds (i.e. singularities of the exponential map of the normal bundle) and the possible types of contact between these submanifolds and spheres have been first studied by Porteous, see [11] and [12].

The intra- and inter-surface level bifurcation sets $\mathcal{S}_{i}$ and $\mathcal{S}_{i, j}$ are loci of centres of bi-tangent spheres touching $X=\bigcup X_{i}$ in two distinct points. If both points of tangency lie on a single surface $X_{i}$ then the centre belongs to $\mathcal{S}_{i}$, otherwise it belongs to $\mathcal{S}_{i, j}$. Clearly, the distance-squared function from a centre of a bi-tangent sphere has the two points of tangency as its critical points, and the corresponding critical values are given by the square of the radius of the bi-tangent sphere. The locus of centres of bi-tangent spheres of a hypersurface is known as symmetry set in the differential geometry literature, and the singularities of such symmetry sets of plane curves and of surfaces in 3-space have been classified by Bruce, Giblin and Gibson [4]. (In the pattern recognition literature, the symmetry set of a plane curve 
is also known under the names skeleton, medial axis and symmetric axis transform.)

Using these geometrical descriptions of the local bifurcation sets $\mathcal{E}_{i}$ and of the level-bifurcation sets $\mathcal{S}_{i}$ and $\mathcal{S}_{i, j}$, we can now define the bifurcation set of the distance-squared functions for arrangements of algebraic hypersurfaces given as zero-sets $X_{i}=h_{i}^{-1}(0)$. Below, $V \| W$ denotes the condition that the pair of vectors $V, W$ in $\mathbb{R}^{d}$ is parallel (obviously, this condition involves the vanishing of $d-1$ functions involving the components of the vectors), and $S(p, x, r):=\|x-p\|^{2}-r^{2}$ defines a $(d-1)$-sphere with centre $p$ and radius $r$. The fact that (at least) one of the principal curvatures of $X_{i}$ at $x$ is equal to $1 / r$ is equivalent to the vanishing of the following two equations:

$$
Q_{i}(x, u):=\operatorname{det}\left(\begin{array}{cc}
\left(d^{2} h_{i}(x)-u \cdot I\right) & d h_{i}(x) \\
\left(d h_{i}(x)\right)^{t} & 0
\end{array}\right)
$$

(where $I$ denotes the $d \times d$ identity matrix) and

$$
R_{i}(x, u, r):=u^{2} r^{2}-\left\|d h_{i}(x)\right\|^{2} .
$$

(The condition $Q_{i}=R_{i}=0$ can be easily deduced from the standard formula for the principal curvatures of a hypersurface defined as zero-set; see, e.g., p. 204 of Spivak [17]. Note that the derivation of this formula on pp. 202-204 of $[17]$ is for hypersurfaces in 3 -space, but the $d$-dimensional case $(d \geq 2)$ is analogous.)

Using this notation, the local bifurcation sets (evolutes) are defined as follows

$$
\begin{gathered}
\mathcal{E}_{i}:=\left\{p \in \mathbb{R}^{d}: \exists x, u, r: \quad h_{i}(x)=S(p, x, r)=Q_{i}(x, u)=R_{i}(x, u, r)=0,\right. \\
d h_{i}(x) \| d S(p, x, r)
\end{gathered}
$$

The level bifurcation sets (symmetry sets) are given by

$$
\left.\begin{array}{c}
\mathcal{S}_{i}:=\operatorname{cl}\left\{p \in \mathbb{R}^{d}: \exists x_{1} \neq x_{2}: \quad h_{i}\left(x_{k}\right)=0, d h_{i}\left(x_{k}\right) \|\left(x_{k}-p\right), k=1,2 ;\right. \\
\left\|x_{1}-p\right\|^{2}=\left\|x_{2}-p\right\|^{2}
\end{array}\right\}
$$

and

$$
\left.\begin{array}{c}
\mathcal{S}_{i, j}:=\left\{p \in \mathbb{R}^{d}: \exists x_{i}, x_{j}: \quad h_{k}\left(x_{k}\right)=0, d h_{k}\left(x_{k}\right) \|\left(x_{k}-p\right), k=i, j ;\right. \\
\left\|x_{i}-p\right\|^{2}=\left\|x_{j}-p\right\|^{2}
\end{array}\right\} .
$$

The estimates in Propositions 2.1, 2.3 and 2.4 for arrangements of parameterized surfaces, in terms of $n$ and $\delta$, have the following analogues, (i)-(iii) of 3.1.1, in the case of $(d-1)$-dimensional zero-sets. 
3.1.1 Proposition. Let $\mathcal{B}$ denote the bifurcation set of the family of distance-squared functions on a collection $X=\bigcup_{i=1}^{n} X_{i}$ of algebraic hypersurfaces of maximal degree $\Delta$. Then the following holds: (i) the degree of $\bigcup \partial \mathcal{S}_{i}^{\prime}$ is at most of order $n \cdot \Delta^{d+3}$; (ii) the number of critical points of the distance-squared function from any point $p \in \mathbb{R}^{d} \backslash \bigcup \mathcal{E}_{i}$ to $X$ is at most of order $n \cdot \Delta^{d}$; and (iii) the number of connected regions of $\mathbb{R}^{d} \backslash \mathcal{B}$ is at most of order $n^{2 d} \cdot \Delta^{2 d^{2}}$.

Proof. Using the new definitions of the components of $\mathcal{B}$ above in the proofs of Propositions 2.1, 2.3 and 2.4 yields the desired results.

\subsection{Arrangements of algebraic sets of higher codimension}

Let $X_{i}=h_{i}^{-1}(0)$ be the $m_{i}$-dimensional zero-set of a polynomial map $h_{i}:=$ $\left(h_{i}^{1}, \ldots, h_{i}^{d-m_{i}}\right): \mathbb{R}^{d} \rightarrow \mathbb{R}^{d-m_{i}}$. The distance-squared function from $p$ to $X_{i}$ has an $A_{k}$-singularity at $x$ if and only if there exists a $\left(d-m_{i}\right)$-sphere with centre $p$ having $(k+1)$-point contact with $X_{i}$ at $x$. Algebraically, the order of contact (or intersection multiplicity) between $X_{i}$ and a $\left(d-m_{i}\right)$ sphere, with defining equations $s^{1}(\xi)=\ldots=s^{m_{i}}(\xi)=0$, at $x$ is equal to the dimension of the vector space

$$
\mathbb{R}[\xi] /\left\langle h_{i}^{1}(\xi-x), \ldots, h_{i}^{d-m_{i}}(\xi-x), s^{1}(\xi-x), \ldots, s^{m_{i}}(\xi-x)\right\rangle .
$$

It is easy to see that such a sphere has at least 2-point contact with $X_{i}$ at $x$ if its centre $p$ lies in the normal space $N_{x} X_{i}=x+\operatorname{span}\left\{d h_{i}^{1}(x), \ldots, d h_{i}^{d-m_{i}}(x)\right\}$ of $X_{i}$ at $x$ (this assumes that $x$ is a regular point of $X_{i}$, but the algebraic definition of the intersection multiplicity above is also valid for the singular locus of $X_{i}$ ).

We can now define the local bifurcation set $\mathcal{E}_{i}$ for complete intersections $X_{i}$ and give an estimate for its degree. The point $p$ lies in the normal space of $X_{i}$ at $x$ if $x-p \in \operatorname{span}\left\{d h_{i}^{1}(x), \ldots, d h_{i}^{d-m_{i}}(x)\right\}$ which means that all $\left(d-m_{i}+1\right) \times\left(d-m_{i}+1\right)$ minors of $\left(\begin{array}{c}d h_{i}(x) \\ x-p\end{array}\right)$ have to vanish. Note that only $m_{i}$ of these minors are independent and that each of them has degree $O\left(\Delta_{i}\right)$. Let $\mathcal{M}_{i}:=\left(\mathcal{M}_{i}^{1}, \ldots, \mathcal{M}_{i}^{m_{i}}\right): \mathbb{R}^{2 d} \rightarrow \mathbb{R}^{m_{i}}$ be a polynomial map whose component functions are such independent minors. Let $\varphi_{i}:=$ $\left(h_{i}, \mathcal{M}_{i}\right): \mathbb{R}^{d} \times \mathbb{R}^{d} \rightarrow \mathbb{R}^{d},(p, x) \mapsto \varphi_{i}(p, x)$ denote the $d$-parameter family of polynomials maps of $\mathbb{R}^{d}$, depending on the parameter vector $p$. Using the algebraic definition of the intersection multiplicity, one checks that the simple roots in $x$ of $\varphi_{i}$ correspond to points of $X_{i}$ having 2-point contact with $\left(d-m_{i}\right)$-spheres with centre $p$ through $x$. Roots of higher multiplicity correspond to points $x$ in which the order of contact is at least 3 -point, hence we define

$$
\mathcal{E}_{i}:=\left\{p \in \mathbb{R}^{d}: \exists x: \varphi_{i}(p, x)=\operatorname{det} d \varphi_{i}(p, x)=0\right\}
$$


The product of the degrees of these defining equations of $\mathcal{E}_{i}$ is at most $O\left(\Delta_{i}^{2\left(m_{i}+1\right)}\right)$.

The level bifurcation set of the family of all distance-squared functions to a pair of complete intersections $X_{i}=h_{i}^{-1}(0)$ and $X_{j}=h_{j}^{-1}(0)$ of dimension $m_{i}$ and $m_{j}$ is given by

$$
\mathcal{S}_{i, j}:=\left\{p \in \mathbb{R}^{d}: \exists x, \bar{x}: \varphi_{i}(p, x)=\varphi_{j}(p, \bar{x})=0,\|x-p\|^{2}=\|\bar{x}-p\|^{2}\right\}
$$

and has degree at most $O\left(\Delta_{i}^{m_{i}+1} \Delta_{j}^{m_{j}+1}\right)$. The level bifurcation set of a single set $X_{i}$ is given by

$$
\mathcal{S}_{i}:=\operatorname{cl}\left\{p \in \mathbb{R}^{d}: \exists x \neq \bar{x}: \varphi_{i}(p, x)=\varphi_{i}(p, \bar{x})=0,\|x-p\|^{2}=\|\bar{x}-p\|^{2}\right\}
$$

and has degree at most $O\left(\Delta_{i}^{2\left(m_{i}+1\right)}\right)$. Recall that $\mathcal{S}_{i}$ is the projection of the algebraic set $\tilde{\mathcal{S}}_{i}:=\mathcal{S}_{i}^{\prime} \cup \partial \mathcal{S}_{i}^{\prime}$. The set $\tilde{\mathcal{S}}_{i}$ is the closure of the difference of two algebraic sets $U \backslash V$, where $U$ is the zero-set of the defining equations of $\mathcal{S}_{i}$, omitting the inequations $x \neq \bar{x}$, and where $V$ is defined by the equations of $\mathcal{S}_{i}$ and by $x=\bar{x}$. Hence $\tilde{\mathcal{S}}_{i}=Z(I(U): I(V))$ is an algebraic set of degree at most $\operatorname{deg} U \sim O\left(\Delta_{i}^{2\left(m_{i}+1\right)}\right)$ and its projection $\mathcal{S}_{i}$ is a semi-algebraic subset of an algebraic set of degree $O\left(\Delta_{i}^{2\left(m_{i}+1\right)}\right)$.

Next recall that the boundary $\mathcal{S}_{i}^{\prime}$ of $\tilde{\mathcal{S}}_{i}$ is contained in the diagonal $E:=$ $\{x=\bar{x}\} \subset \mathbb{R}^{2 d}$. The subspace $E$ is linear which implies that $\operatorname{deg} \tilde{\mathcal{S}}_{i} \cap E=$ $\operatorname{deg} \tilde{\mathcal{S}}_{i}$ and that $\mathcal{S}_{i}^{\prime} \subset \tilde{\mathcal{S}}_{i} \cap E$ has degree at most $O\left(\Delta_{i}^{2\left(m_{i}+1\right)}\right)$.

Finally note that the number of critical points of the distance-squared function from some fixed point $p \in \mathbb{R}^{d} \backslash \mathcal{E}_{i}$ is finite and bounded above by the degree of the map $\varphi_{i}$, which is $O\left(\Delta_{i}^{m_{i}+1}\right)$. Summing up we have the following

3.2.1 Proposition. Let $\mathcal{B}$ denote the bifurcation set of the family of distance-squared functions on a collection $X=\bigcup_{i=1}^{n} X_{i}$ of algebraic sets of maximal degree $\Delta$ and maximal dimension $m$. Then the following holds: (i) the degree of $\bigcup \partial \mathcal{S}_{i}^{\prime}$ is at most of order $O\left(\Delta^{2(m+1)}\right)$; (ii) the number of critical points of the distance-squared function from any point $p \in \mathbb{R}^{d} \backslash \bigcup \mathcal{E}_{i}$ to $X$ is at most of order $n \cdot \Delta^{m+1}$; and (iii) the number of connected regions of $\mathbb{R}^{d} \backslash \mathcal{B}$ is at most of order $n^{2 d} \cdot \Delta^{2(m+1) d}$.

Note that the estimates (ii) and (iii) yield in the case of hypersurfaces ( $m=d-1$ ) the same estimates as in Proposition 3.1.1 (ii) and (iii). It is also interesting to compare these estimates for arrangements of zero-sets with the corresponding bounds in the special case of parameterized $m_{i}$-surfaces given in Section 2. Not surprisingly, the combinatorial complexities (fixing the degrees $\Delta$ or $\delta$ ) are the same. However, in terms of algebraic complexity, the estimates in Propositions 2.1, 2.3 and 2.4 for arrangements of parameterized surfaces are sharper than the corresponding ones in Proposition 3.2.1. This can be seen using the following fact. 
3.2 Lemma. The degree $\Delta_{i}$ of a parameterized $m_{i}$-surface $X_{i}$ given by

$$
x \mapsto X_{i}(x):=\left(X_{i}^{1}(x), \ldots, X_{i}^{d}(x)\right), \quad \delta_{i}:=\sup _{j} \operatorname{deg} X_{i}^{j}
$$

is of order $\delta_{i}^{m_{i}}$ (which implies, for arrangements of such surfaces, that $\left.\Delta \sim O\left(\delta^{m}\right)\right)$.

Proof. Let $\mathcal{L}$ be a $\left(d-m_{i}\right)$-dimensional linear subspace of $\mathbb{R}^{d}$ not contained in $X_{i}$, and let $\mathcal{L}$ be given as zero-set of some linear map $L=$ $\left(L_{1}, \ldots, L_{m_{i}}\right): \mathbb{R}^{d} \rightarrow \mathbb{R}^{m_{i}}$. By Bezout's theorem, $L \circ X_{i}: \mathbb{R}^{m_{i}} \rightarrow \mathbb{R}^{m_{i}}$ has at most $\delta_{i}^{m_{i}}$ roots (counting multiplicities, complex roots and roots at infinity), hence $\left|\mathcal{L} \cap X_{i}\right| \sim O\left(\delta_{i}^{m_{i}}\right)$.

\section{Determining the connected regions in the com- plement of $\mathcal{B}$}

In the present section we sketch the steps of an exact symbolic algorithm for computing the connected regions of $\mathbb{R}^{d} \backslash \mathcal{B}$ for arrangements of algebraic sets defined by polynomials with rational coefficients. This algorithm is analogous to certain substeps of the view graph algorithms for algebraic and semi-algebraic surfaces in [14] and [15], respectively. Roughly speaking, the view graph is the dual of the bifurcation set of the family of all projections of a surface onto planes - the similarity of the algorithms for these bifurcation problems is therefore not too surprising. The bifurcation set $\mathcal{B}$ of the family of distance-squared functions between points $p \in \mathbb{R}^{d}$ and a collection of algebraic sets is a semi-algebraic set which is the projection of a real algebraic set $\tilde{\mathcal{B}} \subset \mathbb{R}^{d} \times \mathbb{R}^{a}$, where $a \leq 2 m$ (for parameterized $m_{i}$-surfaces, $m:=\sup m_{i}$ ) or $a=2 d$ (for zero-sets). The defining equations of the components of $\tilde{\mathcal{B}}$ are polynomials with rational coefficients, and the main steps of the algorithm can be best explained by considering the following diagram:

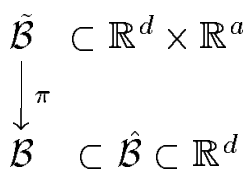

1. Eliminate $x^{1}, \ldots, x^{a}$ between the defining equations of $\tilde{\mathcal{B}}$. Result: the defining equations of the real algebraic set $\hat{\mathcal{B}} \subset \mathbb{R}^{d}$.

2. Decompose $\mathbb{R}^{d}$ into connected regions (of constant combinatorial complexity) such that each such region lies in a single component of $\mathbb{R}^{d} \backslash \hat{\mathcal{B}}$ (and hence of $\mathbb{R}^{d} \backslash \mathcal{B}$ ). 
3. Optional steps: (i) merge regions that lie in a single connected component of $\mathbb{R}^{d} \backslash \hat{\mathcal{B}}$ and (ii) merge regions that are separated by a "branch" of $\hat{\mathcal{B}} \backslash \mathcal{B}$.

REMARKS. (i) In practice it is better to carry out the elimination step for each component $\tilde{\mathcal{B}}_{l}, 1 \leq l \leq 2 n+\left(\begin{array}{c}n \\ 2\end{array}\right)$, separately. For parameterised surfaces one has to eliminate $m_{i}\left(\right.$ for $\left.\tilde{\mathcal{B}}_{l}=\tilde{\mathcal{E}}_{i}\right), 2 m_{i}\left(\right.$ for $\tilde{\mathcal{B}}_{l}=\tilde{\mathcal{S}}_{i}$ ) or $m_{i}+m_{j}$ variables (for $\tilde{\mathcal{B}}_{l}=\tilde{\mathcal{S}}_{i, j}$ ). During the elimination, which can use either multi-polynomial resultants (see, for example, [5]) or Gröbner bases, one can remove repeated factors, because the later steps of the algorithm only require information about the radicals of the elimination ideals $I\left(\hat{\mathcal{B}}_{l}\right):=$ $I\left(\tilde{\mathcal{B}}_{l}\right) \cap \mathbb{Q}[p]$. (Note that the worst case computation time is $D^{O(v)}$ for the multi-polynomial resultant and $D^{2^{O(v)}}$ for Gröbner bases, where $D \leq \delta$ or $\Delta$ is the maximal degree of the input polynomials and $v=d+a$ the number of variables.)

(ii) Suppose that the set $\hat{\mathcal{B}}$ is the union of the zero-sets of $N$ polynomials of maximal degree $D$ and maximal bit length $L$. (Note that $N \sim O\left(n^{2}\right)$ and that $D \sim O\left(\delta^{2 m+1}\right)$ or $O\left(\Delta^{2(m+1)}\right)$.) Here is a (far from complete) list of algorithms for determining regions in the complement of $\mathbb{R}^{d} \backslash \hat{\mathcal{B}}$ :

- The cylindrical algebraic decomposition of Collins [7] yields at most $(N D)^{2^{d}} d$-cells in the complement of $\mathbb{R}^{d} \backslash \hat{\mathcal{B}}$. The cells a re diffeomorphic to open $d$-cubes (so that the number of lower dimensional cells in their closure is independent of $N$ ) and can be determined in $L^{3}(N D)^{2^{d}}$ time.

- Grigor'ev and Vorobjov [9] describe a singly exponential stratification into at most $(N D)^{d^{2}} d$-cells which takes $L^{O(1)}(N D)^{O\left(d^{2}\right)}$ time. It is not clear whether the number of lower dimensional cells in the closure of such a $d$-cell is independent of $N$.

- Chazelle et al. [6] (see also Theorem 8.23 in the book of Sharir and Agarwal [16]) describe a stratification which yields $d$-cells whose closure contains a number of lower dimensional cells which does not depend on $N$. Assuming that the maximal degree $D$ and the bit lengths of all polynomials arising during the computation are bounded by some constant, this stratification consists of at most $O\left(N^{2 d-3+\epsilon}\right)$ cells which can be determined deterministically in $O\left(N^{2 d+1}\right)$ time or by a randomized algorithm in $O\left(N^{2 d-3+\epsilon}\right)$ (expected) time (here $\epsilon$ denotes an arbitrarily small positive constant). Furthermore, given some point $p \in \mathbb{R}^{d}$, the cell containing $p$ can be determined in $O(\log N)$ time. The drawback of this stratification procedure is that, considering the degree $D$ as a variable, the number of cells and the running time become doubly exponential in $d$ with base $D$.

- The complement of $\mathbb{R}^{d} \backslash \hat{\mathcal{B}}$ (and of $\mathbb{R}^{d} \backslash \mathcal{B}$ ) consists of at most $O\left((N D)^{d}\right)$ regions. The algorithms in Rieger $[14,15]$ can be adapted to determine 
these regions. However, such a region will in general contain $O(N)$ "components" of $\hat{\mathcal{B}}$ in its closure.

It would be interesting to reduce the $D^{2^{d}}$-term in the complexity of Chazelle et al. [6] to something singly exponential - or to check whether the cells produced by the singly exponential stratification procedure of Grigor'ev and Vorobjov [9] contain a number of lower dimensional cells in their closure which is independent of $N$. The 2-D examples shown in the next section are based on the cylindrical algebraic decomposition of Collins.

The above decompositions of $d$-space into regions in the complement of the bifurcation set can be used to solve the following proximity problems exactly (i.e. without numerical errors). Given a point $p \in \mathbb{R}^{d}$ and a set of defining polynomials of $X$ with rational coefficients we would like to do the following:

1. find the $k$ nearest sets $X_{i}$;

2. find the nearest point in $X$;

3. and, provided that $X$ is compact, find the farthest point in $X$ (and hence the smallest sphere with centre $p$ enclosing $X$ ).

For all three problems we first decompose $\mathbb{R}^{d}$ into regions which lie in a single connected component in the complement of $\bigcup \mathcal{S}_{i, j}$ (or $\mathcal{B}$ or $\hat{\mathcal{B}}$ - the latter two possibilities yield finer decompositions but with the same "leading term" w.r.t. the asymptotic complexity in the number of regions). For problem 1 we store for each region the $k$ nearest $X_{i}$ (for any sample point in the region), for problem 2 the nearest $X_{i}$ and for problem 3 the farthest. This completes the preprocessing.

Now assume that the coordinates of $p$ are either rational or algebraic numbers $p=\left(\alpha^{1}, \ldots, \alpha^{d}\right)$ (where each $\alpha^{j}$ is encoded by some minimal polynomial $m^{j}(t)=0$ and some isolating interval with rational endpoints). Problem 1 then boils down to finding the region in the decomposition which contains $p$. Using the algorithm in [6], this can be done in $O(\log n)$ time assuming that the degrees and coefficient sizes of the defining polynomials of $X$ and of the minimal polynomials $m^{j}$ are bounded by some constant. For problems 2 and 3 one first determines the region containing $p$, this yields the $X_{i}$ containing respectively the nearest and farthest point $q$ in $X$ from $p$ and takes $O(\log n)$ time. In general, the coordinates of $q$ are algebraic numbers $\left(\beta^{1}, \ldots, \beta^{d}\right)$, and a possible representation of the result of problems 2 and 3 consists of minimal polynomials and isolating intervals of the coordinates of $q$. Once we know the region containing $p$ and hence $X_{i} \ni q$, we can forget all $X_{j}, j \neq i$, so that the combinatorial time complexity of determining $q$ is obviously $O(1)$. However, in terms of algebraic time complexity, the computations described next are quite expensive. From 
the discussion in the earlier sections of this paper we know that the critical points of the distance-squared function from $p$ to $X_{i}$ are either the real roots of $d f_{i}^{1}(p, x)=\ldots=d f_{i}^{m_{i}}(p, x)=0$, where $d f_{i}^{j} \in \mathbb{Q}\left(\alpha^{1}, \ldots, \alpha^{d}\right)[x]$, (for parameterized $m_{i}$-surfaces $\left.X_{i}\right)$ or of $\varphi_{i}^{1}(p, x)=\ldots=\varphi_{i}^{d}(p, x)=0$, where $\varphi_{i}^{j} \in \mathbb{Q}\left(\alpha^{1}, \ldots, \alpha^{d}\right)[x]$, (for zero-sets $\left.X_{i}\right)$. The real roots $\xi_{1}, \ldots, \xi_{s}$ of these systems are isolated and have algebraic coordinates whose minimal polynomials and isolating intervals can be determined by computing a diagonal basis of the systems and by isolating the roots of univariate polynomials with algebraic coefficients (using primitive element methods and the modified Uspenski algorithm). For parameterized surfaces $X_{i}$ we have to determine the root $\xi_{j}, 1 \leq j \leq s$, for which the algebraic number $f_{i}\left(p, \xi_{j}\right)$ is minimal (problem 2) or maximal (problem 3); and the result is $q=X_{i}\left(\xi_{j}\right)$ whose coordinates are algebraic numbers. For zero-sets $X_{i}$ we have to determine the $\xi_{j}$ for which $\left\|\xi_{j}-p\right\|^{2}$ is minimal or maximal; and the result is $q=\xi_{j}$.

REMARK. The exact symbolic computation of the nearest and farthest point $q \in X$ from $p$ could also be replaced by some approximate numerical computation. For example, one could determine one sample point $p^{\prime}$ for each region in the complement of $\mathcal{B}$ (or $\hat{\mathcal{B}}$ ) and the corresponding nearest or farthest point $q^{\prime} \in X$. After determining the region containing $p$ (as before), one knows that any path in this region joining its sample point $p^{\prime}$ with $p$ corresponds to a unique path in $X$ joining the corresponding nearest/farthest points $q^{\prime}$ and $q$. This follows from the fact that the critical points of the distance-squared function are isolated in the complement of $\mathcal{B}$ and the continuity of the map which assigns to $p$ its nearest/farthest point in $X$ (Proposition 2.4). Note that a decomposition into regions in the complement of $\bigcup \mathcal{S}_{i, j}$, which is sufficient for the exact symbolic method described above, would not have this property and would be too coarse for a numerical procedure which traces the curve from $q^{\prime}$ to $q$. The combinatorial complexities of such a numerical method and the symbolic method above are the same but the algebraic complexity of the curve tracing procedure, using some sufficiently large step size, should be much smaller.

\section{Some examples for arrangements in the plane}

The first example in Figure 1 consists of a pair of parabolas $X_{1}(x)=\left(x, x^{2}-\right.$ $1), X_{2}(x)=\left(x, 1-x^{2}\right)$. The sets $\hat{\mathcal{E}}_{1}$ and $\hat{\mathcal{E}}_{2}$ are cubic curves with a cusp singularity, and it turns out that in this example $\hat{\mathcal{E}}_{i}=\mathcal{E}_{i}, i=1,2$. The sets $\hat{\mathcal{S}}_{1}$ and $\hat{\mathcal{S}}_{2}$ are vertical lines through the origin, and the sets $\mathcal{S}_{i}$ are half-lines "inside" the cusp curves $\mathcal{E}_{i}$. The set $\hat{\mathcal{S}}_{1,2}$ consists of a horizontal line through the origin and the zero-set $Z$ of an irreducible (over $\mathbb{Q}$ ) degree 12 polynomial. The set $Z$ has three real components: a compact curve with 6 cusps and a pair of non-singular curves passing through the intersection points of the parabolas. Figure 2 shows a cylindrical algebraic decomposition of of the 


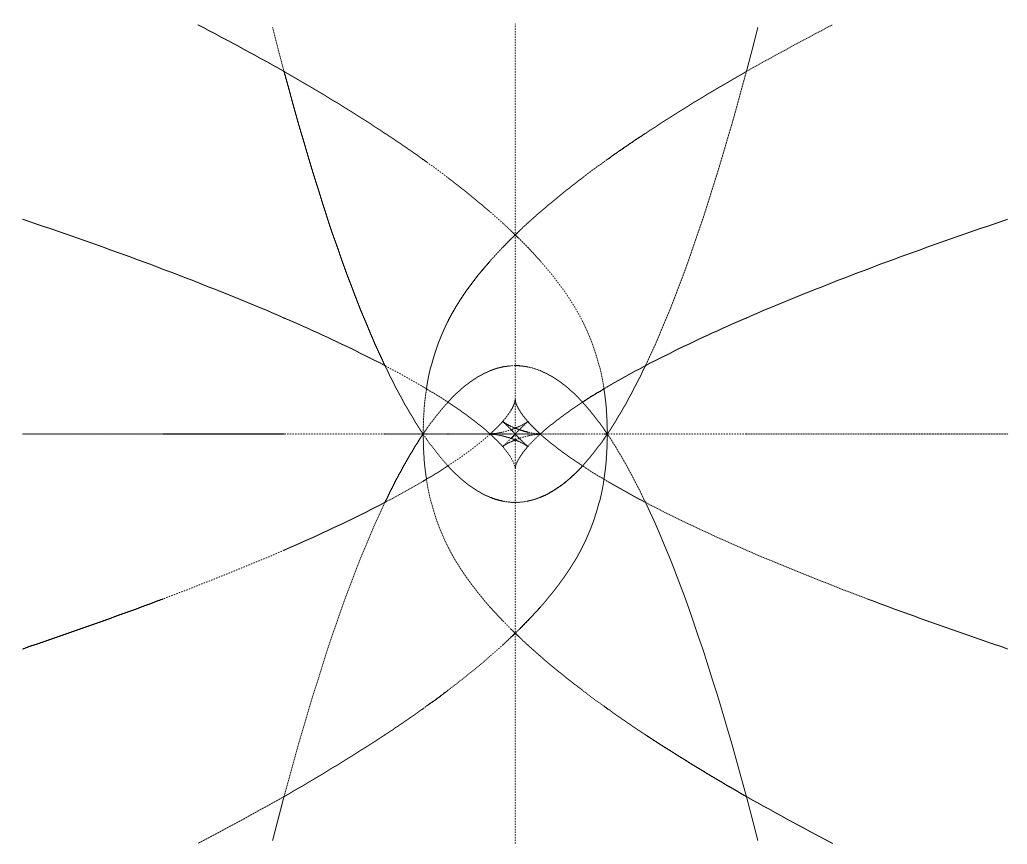

Figure 1: The set $\hat{\mathcal{B}}$ for a pair of parabolas.

plane into regions in the complement of $\mathbb{R}^{2} \backslash \hat{\mathcal{B}} \cup X_{1} \cup X_{2}$, which are arranged in "vertical cylinders". The cylinders are bounded by vertical tangent lines or by vertical lines passing through singular points. The regions within a cylinder $I \times \mathbb{R}$, where $I$ is an interval on the $x$-axis, are separated by nonintersecting function graphs over $I$.

Figure 3 shows the set $\hat{\mathcal{S}}_{1,2}$ for a parabola $X_{1}(x)=\left(x, x^{2}\right)$ and a point $X_{2}=(1,2)$. Note that the Voronoi diagram of $X_{1}, X_{2}$ consists of just two regions: the region cut out by $\hat{\mathcal{S}}_{1,2}$ containing the point $X_{2}$ and the complement of the closure of this region. The curve $\hat{\mathcal{S}}_{1,2}$ has 2 cusps, which correspond to centres of osculating circles of $X_{1}$ which pass through the point $X_{2}$. Figure 4, which also shows the set $\hat{\mathcal{E}}_{1}$ which contains the evolute, illustrates this fact: the cusps of $\hat{\mathcal{S}}_{1,2}$ lie on the evolute (and hence are centres of osculating circles).

\section{Regions of $\mathbb{R}^{d} \backslash \mathcal{B}$ and Voronoi regions}

Most works on Voronoi diagrams of (semi)-algebraic sets assume that the degrees of these sets are bounded by some constant and study the complexity of the Voronoi diagram as a function of $n$ and $d$ only. In the present section we shall therefore assume that the degrees of the algebraic sets in an arrangement are bounded above by some constant and compare the combinatorial complexities of $\mathbb{R}^{d} \backslash \mathcal{B}$ and of $k$ th-order Voronoi diagrams of such arrangements. 


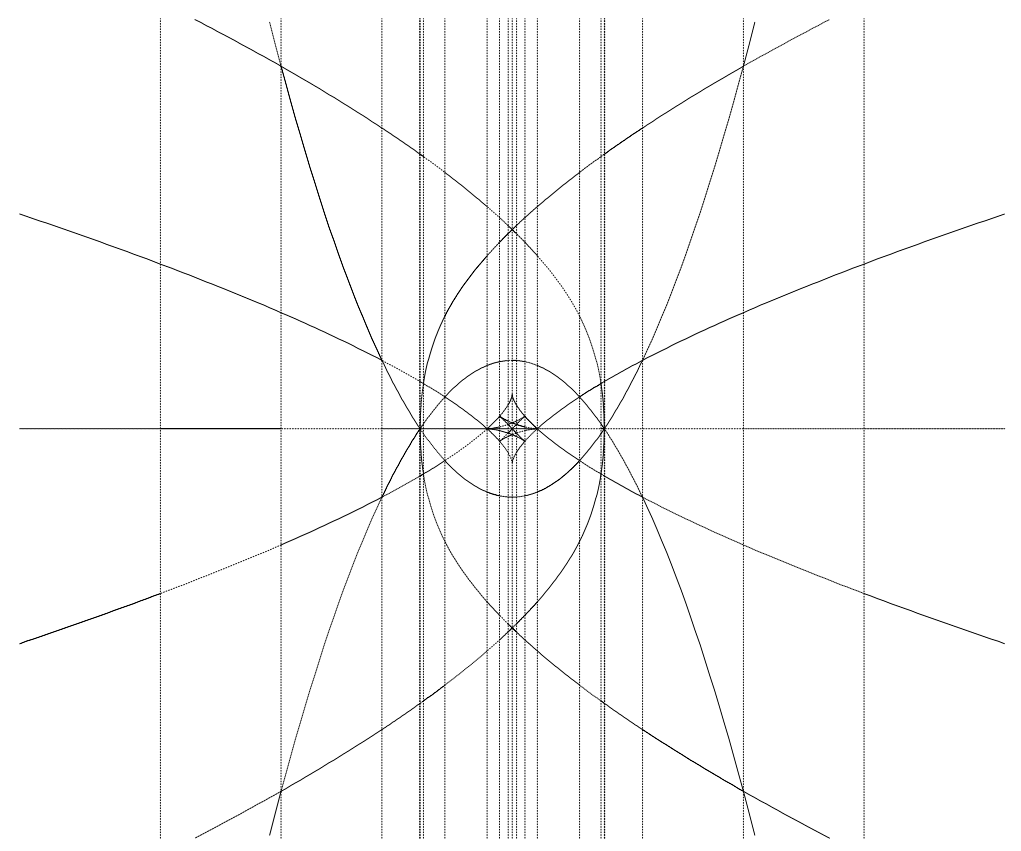

Figure 2: Cylindrical algebraic decomposition of $\hat{\mathcal{B}}$ and a pair of parabolas.

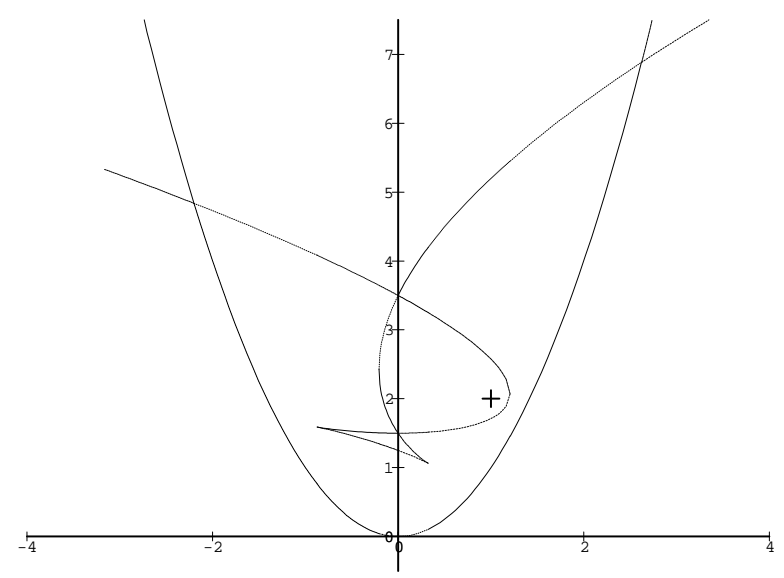

Figure 3: The set $\hat{\mathcal{S}}_{1,2}$ for a parabola $X_{1}$ and the point $X_{2}=(1,2)$ (marked by a cross). 


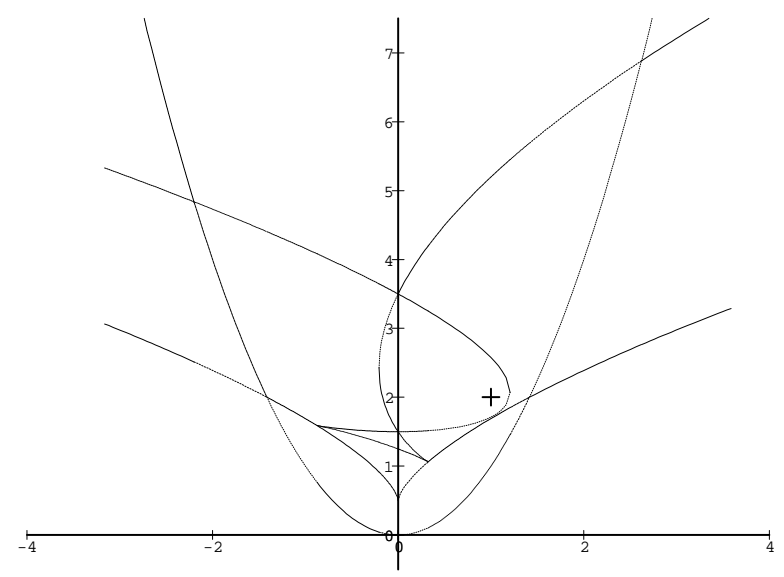

Figure 4: The sets $\hat{\mathcal{S}}_{1,2}$ and $\hat{\mathcal{E}}_{1}$ for a parabola $X_{1}$ and the point $X_{2}=(1,2)$.

Sharir and Agarwal show in Appendix 7.1 of [16] that the first-order Voronoi diagram of $n$ disjoint convex semi-algebraic sets of "constant description size" (i.e. defined by $O(1)$ polynomial (in)equations of bounded degree and coefficient size) in $d$-space has $O\left(n^{d+\epsilon}\right)$ regions (for any $\epsilon>0$ ). An open problem, in this context, is to prove or disprove that there are actually at most $O\left(n^{d-1}\right)$ such regions. In the plane $(d=2)$, and under slightly different hypotheses, such a sharper bound has been established in the following work. Alt and Schwarzkopf [1] study the first-order Voronoi diagram of $n$ points and parameterized algebraic curve segments in the plane which has $O(n)$ regions and can be constructed by a randomized algorithm in $O(n \log n)$ (expected) time. Their algorithm concentrates on the combinatorial aspect of the problem and assumes that the semi-algebraic level bifurcation sets $\mathcal{S}_{i}$ and $\mathcal{S}_{i, j}$ (in our notation) can be determined by some (numerical) polynomial equation solver. We have seen in the previous couple of sections that the bifurcation sets $\mathcal{S}_{i}$ and $\mathcal{S}_{i, j}$ can also be determined symbolically (i.e. by exact methods).

In terms of combinatorial complexity there are at most $O\left(n^{2 d}\right)$ connected regions of $\mathbb{R}^{d} \backslash \mathcal{B}$, i.e. many more regions than Voronoi regions. Note, of course, that the constancy of the number of critical points of a collection of distance-squared functions and of the ordering of these critical points (expressed by the invariance of the indices $\nu_{1}, \ldots, \nu_{c}$ ) within a region of $\mathbb{R}^{d} \backslash \mathcal{B}$ is much more than is required for supporting efficient nearest-point queries. For such queries it suffices to stratify $\mathbb{R}^{d}$ into connected regions in which $\nu_{1}$ is invariant (and, for certain numerical applications, $p \mapsto \xi_{1, \nu_{1}}(p)$ 
is continuous).

Even the problems of finding the $k$-nearest sets $X_{i}$ (for $1 \leq k \leq n-$ 1 ) and of finding the farthest point in $X=\bigcup_{i=1}^{n} X_{i}$ (assuming that $X$ is compact) do not require all the invariance properties of the regions of $\mathbb{R}^{d} \backslash \mathcal{B}$. Note, for example, that all critical points corresponding to saddle points are irrelevant for such proximity problems. The classical Critical Point Theorem says that the Euler characteristic of a $m$-manifold is equal to $\sum_{j=0}^{m}(-1)^{j} I_{j}$, where $I_{j}$ is the number of Morse critical points of some generic distance-squared function on $X$ of index $j$ (i.e. whose matrix of second derivatives has $j$ negative eigenvalues) - the critical points of index 0 and $m$ are minima and maxima, all other critical points are saddle points. However, in terms of combinatorial complexity, the number of saddle-points of some distance-squared function on $X$ is $O(n)$. The difference in the combinatorial complexities of $\mathbb{R}^{d} \backslash \mathcal{B}$ and of the first-order (and also the higher-order) Voronoi diagram $-O\left(n^{2 d}\right)$ versus $O\left(n^{d+\epsilon}\right)$ - has other causes (see below).

First, we fix some notation. Let $V_{k}$ denote the boundary of the regions of the $k$ th-order Voronoi diagram and set $Y:=\bigcup_{1<i<j<n} \mathcal{S}_{i, j}$. Denote the locus of centres of $r$-tangent spheres of $X$ by $\mathcal{L}\left(A_{\geq 1}^{r}\right)$ (note that the distancesquared function from such a centre to $X$ has $r^{-} A_{>1}$-singularities) and the locus of centres of $s$ distinct, at least bi-tangent spheres of $X$ by $\mathcal{L}\left({ }_{s} A{ }_{\geq 1}^{\geq 2}\right)$, and let $\mathcal{N}(Y)$ denote the set of non-immersive points of $Y$. The singular locus of $Y$ consists of the following components:

$$
\operatorname{Sing}(Y):=\mathcal{L}\left(A_{\geq 1}^{\geq 3}\right) \cup \mathcal{L}\left({ }_{2} A_{\geq 1}^{\geq 2}\right) \cup \mathcal{N}(Y) \text {. }
$$

Next, we define subsets $Y_{k} \supset V_{k}$ of $Y$ as follows. Let $B(Y)$ denote the set of connected $(d-1)$-dimensional components ("branches") of $Y \backslash \mathcal{L}\left(A_{>1}^{>3}\right)$. Let $L$ be the set of strata of the following stratification of $\mathcal{L}\left(\geq_{2} A_{\geq 1}^{\geq 2}\right)$ : start with the smallest $s>1$ such that $\mathcal{L}\left({ }_{s} A_{\geq 1}^{\geq 2}\right)$ is non-empty and take as strata the connected components of $\mathcal{L}\left({ }_{s} A_{>1}{ }^{2}\right)$ (note: for a generic arrangement $X, s=d$ yields point-strata), then, for $s-1 \leq i \leq 2$, take as strata the connected components of $\mathcal{L}\left({ }_{i} A_{\geq 1}^{\geq 2}\right) \backslash \mathcal{L}\left({ }_{i+1} A_{\geq 1}^{\geq 2}\right)$. Below, we shall impose certain conditions on $X$ which ensure that the stratification $L$ enjoys certain desirable properties - without such conditions the strata may not even be manifolds. For any $l \in L$, let $l_{k}$ denote the set of branches $b \in B(Y)$ passing through $l$ which correspond to the $k$ smallest bi-tangent spheres with centre in $l$; for $\sup \left\{i: l \subset \mathcal{L}\left({ }_{i} A_{\geq 1}^{\geq 2}\right)\right\}<k$ the set $l_{k}$ contains all $i$ branches through l. We can now define

$$
Y \backslash Y_{k}:=\left\{b \in B(Y): \exists l \in L: l \subset \mathrm{cl} b, \quad b \notin l_{k}\right\} .
$$

The following result sheds some light on the difference in the combinatorial complexities of the sets of regions of $\mathbb{R}^{d} \backslash \mathcal{B}$ and of $k$ th-order Voronoi 
regions.

6.1 Proposition. For any arrangement of parameterized or implicitely defined algebraic sets (whose degrees are bounded by some constant) in dspace consisting of $n$ elements of any positive codimension the following holds:

(i) $V_{k} \subset Y_{k} \subset Y \subset \mathcal{B}, 1 \leq k \leq n-1$.

(ii) $\mathbb{R}^{d} \backslash Y_{k}$ has at most $O\left(\max \left(n^{d+1}, \min \left(n^{2 k}, n^{2 d}\right)\right)\right)$ connected regions.

(iii) The combinatorial complexity of $\mathcal{L}\left({ }_{d} A_{\geq 1}^{\geq 2}\right)$ is $\prod_{j=1}^{d}\left(\begin{array}{c}n \\ 2\end{array}\right) \sim O\left(n^{2 d}\right)$ and represents the "leading term" in the combinatorial complexity of $\mathbb{R}^{d} \backslash \mathcal{B}$.

Proof. (i) simply follows from the definitions of these sets. For the proof of (ii) and (iii) it is convenient to distinguish "generic" and "nongeneric" arrangements $X$, which are defined as follows. Let $\mathcal{X}$ be the space of arrangements $X \subset \mathbb{R}^{d}$ of $n$ zero-sets $X_{i}$ of codimension $c_{i}$ and maximal degree $\Delta$ (or of $n m_{i}$-surfaces $X_{i}$ parameterized by polynomials of degree $\leq \delta)$. $\mathcal{X}$ can be identified with the finite dimensional space of coefficients of $\sum_{i=1}^{n} c_{i}$ polynomials in $d$ variables of degree $\leq \Delta$ (or of $n d$ polynomials in $\sum_{i=1}^{n} m_{i}$ variables of degree $\leq \delta$ ). Now define the following sets

$$
W_{1}:=\left\{X \in \mathcal{X}: \exists r \geq 2: \operatorname{dim} \mathcal{L}\left(A_{\geq 1}^{r}\right)>d-r+1\right\}
$$

and

$$
W_{2}:=\left\{X \in \mathcal{X}: \exists s \geq 1: \operatorname{dim} \mathcal{L}\left({ }_{s} A_{\geq 1}^{\geq 2}\right)>d-s\right\} .
$$

(Note that $X$ denotes both a subset of $\mathbb{R}^{d}$ as well as a point of $\mathcal{X}$, but the meaning of $X$ should be clear from the context.) One shows, using the defining equations of the sets $\mathcal{L}(\cdot)$, that $W_{1} \cup W_{2}$ is a Zariski closed subset of $\mathcal{X}$. We shall therefore say that an element $X$ in $\mathcal{X} \backslash W_{1} \cup W_{2}$ is generic and one in $W_{1} \cup W_{2}$ non-generic.

First, assume that $X$ is generic. Consider the following stratification of $d$-space. As 0 -dimensional strata we either take $\mathcal{L}\left(A_{\geq 1}^{d+1}\right) \cup \mathcal{L}\left({ }_{d} A_{\geq 1}^{\geq 2}\right)$ (for $k \geq d$ ) or just $\mathcal{L}\left(A_{\geq 1}^{d+1}\right)$ (for $k<d$ ). The strata of dimension $1 \leq d-i \leq d-2$ are either

$$
\mathcal{L}\left(A_{\geq 1}^{i+1}\right) \cup \mathcal{L}\left({ }_{i} A_{\geq 1}^{\geq 2}\right) \backslash \mathcal{L}\left(A_{\geq 1}^{i+2}\right) \cup \mathcal{L}\left({ }_{i+1} A_{\geq 1}^{\geq 2}\right)
$$

(for $k \geq i$ ) or

$$
\mathcal{L}\left(A_{\geq 1}^{i+1}\right) \backslash \mathcal{L}\left(A_{\geq 1}^{i+2}\right)
$$


(for $k<i$ ). Finally, we take either $Y_{k} \backslash \mathcal{L}\left(A_{\geq 1}^{3}\right) \cup \mathcal{L}\left({ }_{2} A_{\geq 1}^{\geq 2}\right)$ (for $k \geq 2$ ) or $Y_{k} \backslash \mathcal{L}\left(A_{>1}^{3}\right)$ (for $k=1$ ) as $\left(d-1\right.$ )-dimensional strata, and $\mathbb{R}^{d} \backslash Y_{k}$ as $d$-dimensional strata. Letting $e_{i}$ denote the number of $i$-dimensional strata of this stratification, we want to estimate $e_{d}$. The combinatorial complexity of $\mathcal{L}\left(A_{\geq 1}^{r}\right)$ is $\left(\begin{array}{l}n \\ r\end{array}\right)$ and that of $\mathcal{L}\left({ }_{s} A_{\geq 1}^{\geq 2}\right)$ is $\prod_{i=1}^{s}\left(\begin{array}{c}n \\ 2\end{array}\right) \sim O\left(n^{2 s}\right)$. Taking a 1-point compactification $S^{d}=\mathbb{R}^{d} \cup\{\infty\}$ and considering the induced stratification in the $d$-sphere, we see that the alternating sum of the $e_{i}$ is equal to the Euler characteristic $\chi\left(S^{d}\right)=1+(-1)^{d}$. The combinatorial complexities of the sets $\mathcal{L}(\cdot)$ above imply that, for this induced stratification, $e_{i} \sim O\left(\max \left(n^{d+1}, \min \left(n^{2 k}, n^{2 d}\right)\right)\right)$ for $0 \leq i \leq d-1$. It now follows that $e_{d}$ is bounded above by $O\left(\max \left(n^{d+1}, \min \left(n^{2 k}, n^{2 d}\right)\right)\right)$, which implies statement (ii). Under the hypothesis that $X$ is generic, there are no points in the bifurcation set $\mathcal{B}$ which are centres of more than $d$ distinct bi-tangent spheres of $X$. One checks that $\mathcal{L}\left({ }_{d} A_{\geq 1}^{\geq 2}\right)$ is a subset of strata of $\mathcal{B}$ having the highest combinatorial complexity, namely $O\left(n^{2 d}\right)$, which implies (iii).

For non-generic arrangements $X \in W_{1} \cup W_{2}$, we consider a "linear deformation" $X_{t}, t \in(-\epsilon,+\epsilon)$, of $X=X_{0}$ such that $X_{0}$ is the only non-generic element - linear in the sense that $t \mapsto X_{t}$ defines a line in the space of coefficients which can be identified with $\mathcal{X}$. (Such a deformation can be obtained, for example, by constructing a stratification of the semi-algebraic set $W_{1} \cup W_{2}$ and by restricting a line in the normal space of the stratum containing $X$ to some sufficiently small open neighborhood.) Consider the union $U$ of any of the semi-algebraic sets $U_{t}=\mathcal{B}_{t}$ or $Y_{t}$ or $\left(Y_{k}\right)_{t}$ associated to $X_{t}, U$ is a semi-algebraic subset of $\mathbb{R}^{d} \times(-\epsilon,+\epsilon)$. We claim that the combinatorial complexity of the number of regions in the complement of $U_{t}$ is lower semi-continuous in $t$, which implies that the bounds in the generic case $(t \neq 0)$ are also valid in the non-generic case $(t=0)$. This claim follows from the following argument. Let $\epsilon>0$ be small enough such that $U$ is transverse to all hyperplanes $t=c$, for any constant $|c|<\epsilon$, except $t=0$ ( $U$ is a, in general, singular semi-algebraic set, and transverse means that the hyperplane in question is transverse to all the strata of a suitable stratification of $U$, e.g. a stratification satisfying the Whitney condition $(b)$ ). So the number of regions in the complement of $U_{t}$ are locally constant for $t \in(-\epsilon, 0)$ and $t \in(0, \epsilon)$, denote these numbers by $r_{-}$and $r_{+}$respectively. Setting $H_{ \pm}:=\mathbb{R}^{d} \times( \pm \epsilon, 0)$, the number of regions of $H_{ \pm} \backslash U \cap H_{ \pm}$is equal to $r_{ \pm}$. Finally, note that the number of regions of $\mathbb{R}^{d} \backslash U_{0}$ is less than or equal to the number of regions of $H_{ \pm} \backslash U \cap H_{ \pm}$(the former regions being contained in the closures of the latter).

REMARK. In the case where $k$ is bounded by some constant (independent of $d$ ), the bound in 6.1 for the number of regions of $\mathbb{R}^{d} \backslash Y_{k}$ is unlikely to yield a reasonably tight bound for the number of $k$ th-order Voronoi regions. The bounds in [1] and in [16] for $k=1$ (and slightly different "sites" in the 
arrangement $X$ ) suggest that the $n^{d+1}$-term could be lowered to $n^{d}$ or even $n^{d-1}$. The obstruction to an $O\left(n^{d}\right)$ bound for the number of regions in the complement of $Y_{1}$ (and hence of $V_{1} \subset Y_{1}$ ) is the combinatorial complexity of $\mathcal{L}\left(A_{\geq 1}^{d+1}\right)$, and to an $O\left(n^{d-1}\right)$-bound that of both $\mathcal{L}\left(A_{\geq 1}^{d+1}\right)$ and $\mathcal{L}\left(A_{\geq 1}^{d}\right)$. Note, for example, that

$$
\mathcal{L}\left(A_{2 m}^{d+1}\right) \cup\left\{p \in \mathcal{L}\left(A_{2 m+1}^{d+1}\right): X \cap B(p) \neq \emptyset\right\} \not \subset V_{1},
$$

where $B(p)$ denotes the interior of the $(d+1)$-tangent sphere with centre $p$. It would be good to know whether the combinatorial complexity of $\mathcal{L}\left(A_{\geq 1}^{d+1}\right) \cap V_{1}$ is of order $n^{d}$.

\section{Acknowledgements}

I am very grateful to the research group AG1 at the Max-Planck-Institut in Saarbrïcken for its hospitality and financial support.

\section{References}

[1] H. Alt and O. Schwarzkopf, "The Voronoi diagram of curved objects", Proc. 11th Annual Symp. Comput. Geometry, Vancouver, B.C. Canada (ACM, 1995), 89-97

[2] R. Benedetti and J.-J. Risler, Real algebraic and semi-algebraic sets, (Hermann, Paris, 1990)

[3] J.W. Bruce, "Lines, circles, focal and symmetry sets", Math. Proc. Cambridge Phil. Soc. 118 (1995), 411-436

[4] J.W. Bruce, P.J. Giblin and C.G. Gibson, "Symmetry sets", Proc. Royal Soc. Edinburgh 101A (1985), 163-186

[5] J. Canny, "Generalised characteristic polynomials", J. Symbolic Computation 9 (1990), 241-250

[6] B. Chazelle, H. Edelsbrunner, L. Guibas and M. Sharir, "A singly exponential stratification scheme for real semi-algebraic varieties and applications", Theoret. Comput. Sci. 84 (1991), 77-105

[7] G.E. Collins, "Quantifier elimination for real closed fields by cylindrical algebraic decomposition", Proc. 2nd GI Conf. Automata Theory and Formal Languages, Springer LNCS 33, (Springer Verlag, BerlinHeidelberg-New York, 1975), 134-183

[8] H. Edelsbrunner and R. Seidel, "Voronoi diagrams and arrangements", Discrete Comput. Geom. 1 (1986), 25-44 
[9] D.Yu. Grigor'ev and N.N. Vorobjov, "Solving systems of polynomial inequalities in subexponential time", J. Symbolic Computation 5 (1988), $37-64$

[10] J. Milnor, "On the Betti numbers of real varieties", Proc. Amer. Math. Soc. 15 (1964), 275-280

[11] I.R. Porteous, "The normal singularities of a submanifold", J. Differential Geom. 5 (1971), 543-564

[12] I.R. Porteous, "The normal singularities of surfaces in $\mathbb{R}^{3 "}$, Proc. Symposia in Pure Math. Vol. 40 (1983), Part 2, (American Mathematical Society, Providence RI), 379-393

[13] I.R. Porteous, "Probing singularities", Proc. Symposia in Pure Math. Vol. 40 (1983), Part 2, (American Mathematical Society, Providence RI), 395-406

[14] J.H. Rieger, "Computing view graphs of algebraic surfaces", J. Symbolic Computation 16 (1993), 259-272

[15] J.H. Rieger, "On the complexity and computation of view graphs of piecewise smooth algebraic surfaces", Phil. Trans. R. Soc. Lond. Ser. A, (in press)

[16] M. Sharir and P.K. Agarwal, Davenport-Schinzel sequences and their geometric applications, (Cambridge University Press, 1995)

[17] M. Spivak, A comprehensive introduction to differential geometry, Vol. III, (Publish or Perish Inc., Berkeley, 1979, 2nd Edition) 